\title{
Ritmo dos jogos das finais das competições europeias de basquetebol (1988-2006) e as estatísticas que discriminam os jogos mais rápidos dos jogos mais lentos
}

\author{
Jorge Malarranha ${ }^{1}$ \\ Jaime Sampaio² \\ https://doi.org/10.5628/rpcd.07.02.202
}

\author{
${ }^{1}$ Comissão Instaladora dos Ensinos \\ na Área da Saúde e do Bem Estar \\ Universidade de Évora \\ ${ }^{2}$ Departamento de Desporto \\ Universidade de Trás-os-Montes e Alto Douro
}

\section{RESUMO}

O objectivo deste estudo foi caracterizar o ritmo dos jogos de Basquetebol, medido pelo número de posses de bola, e identificar diferenças nas variáveis estatísticas entre os jogos realizados a ritmos mais elevados e os jogos realizados a ritmos mais reduzidos. Os dados utilizados reportam-se aos registos estatísticos colectivos dos jogos das finais das três competições europeias de clubes referentes às épocas entre 1987-88 e 2005-06 (Euroliga, ULEBCUP e FIBACUP, $\mathrm{n}=27$ ). Foram constituídos dois grupos de análise a partir do número de posses de bola por jogo: jogos mais rápidos e jogos mais lentos. Os resultados sugerem uma tendência para um aumento do número de posses de bola e pontos marcados e uma diminuição da eficácia ofensiva nos jogos analisados. Por outro lado, a eficácia ofensiva dos jogos mais lentos foi significativamente superior à eficácia ofensiva dos jogos mais rápidos $(p 0,05)$. A função discriminante obtida foi baseada na análise dos coeficientes canónicos estruturais (CCE) e identificou as faltas cometidas $(\mathrm{CCE}=0,44)$, as faltas sofridas $(\mathrm{CCE}=0,43)$, os ressaltos defensivos $(\mathrm{CCE}=0,38)$ e os lançamentos livres falhados $(\mathrm{CCE}=0,37)$ como as estatísticas que melhor discriminam os jogos mais lentos dos mais rápidos $(p \leq 0,05)$. Os treinadores podem utilizar estes resultados na selecção dos jogadores e na preparação das suas equipas, decidindo o ritmo de jogo a impor durante os jogos e os jogadores mais adequados para desempenhar essas tarefas.

\begin{abstract}
Basketball game rhythm in the European Competitions Finals' (1988-2006) and the game-related statistics that discriminate between fast and slow paced games

The aim of this study was to characterize Basketball game rhythm, as measured by ball possessions, and to identify the differences in gamerelated statistics between high and low rhythm games. Archival data report to team game-related statistics from the final games of the three European competitions for clubs from 1987-88 to 2005-06 season (Euroleague, ULEBCUP and FIBACUP, $n=27$ ). Two groups were made according to the number of ball possessions per game: fastest games and slowest games. The results seem to suggest an increase in ball possessions per game and a decrease in points per game and offensive rating. Slowest games' offensive ratings were significantly superior to the fastest games' ratings $(p \leq 0,05)$. The analysis of the discriminant function was based on the observation of the structure coefficients $(S C)$, and identified committed fouls $(S C=0,44)$, received fouls $(S C=0,43)$, defensive rebounds $(S C=0,38)$ and unsuccessful freethrows $(S C=0,37)$ as the game-related statistics that best discriminate fastest from slowest games. Coaches can use these results in players' selection and to prepare the team, deciding which game rhythm to impose during games and which players' are best prepared to perform these tasks.
\end{abstract}

Key-words: Basketball, statistics, game rhythm

Palavras-chave: Basquetebol, estatísticas, ritmo de jogo. 


\section{INTRODUÇÃO}

Nos jogos desportivos colectivos, o processo de preparação desportiva das equipas de alto-nível centrase no objectivo de preparar a equipa para ser capaz executar, em situação de competição, acções tácticas que corporizam uma estratégia colectiva para defrontar determinado adversário, no sentido de vencer o jogo. Este processo de preparação é complexo e implica cada vez mais que as equipas técnicas necessitem da contribuição de especialistas nos domínios da análise do jogo, no sentido de melhor caracterizarem os adversários e consequentemente, melhor definirem e prepararem a estratégia para os defrontar. Particularmente no jogo de Basquetebol, os estudos que recorrem às estatísticas dos jogos têm sido utilizados e explorados, quer na investigação quer no processo de treino, porque permitem que se realize uma caracterização dos adversários na perspectiva de várias dimensões - táctica, técnica, psicológica, física $(1,2,5,13,14)$.

O controlo do ritmo do jogo, entendido como o controlo da velocidade a que decorre o jogo nas suas diferentes fases (transições defesa-ataque e ataquedefesa, ataque e defesa organizados) é um dos aspectos que os treinadores consideram como mais relevantes para o desfecho final dos jogos ${ }^{(4,9)}$. Contudo, a investigação científica não tem tratado deste problema de acordo com a sua importância. De facto, apenas existe a ideia de que os jogos mais decisivos decorrem a ritmos mais lentos.

No Basquetebol profissional norte-americano, Oliver(4) estudou a variação das posses de bola, pontos marcados e eficácia ofensiva (pontos marcados divididos pelas posses de bola) nos jogos da fase regular referentes às épocas entre 1973 e 1995. Os resultados encontrados revelaram um decréscimo do número de posses de bola e pontos marcados, mas revelam, simultaneamente, um aumento da eficácia ofensiva das equipas. O número de posses de bola é uma medida válida do ritmo do jogo(4). Este autor identificou também uma diminuição do número de perdas de bola e de lançamentos durante os jogos, enquanto que o número de lançamentos livres e de ressaltos ofensivos permanece semelhante.

As referências disponíveis na literatura que tratam destes objectivos procuraram analisar as diferenças entre os valores das posses de bola(que representam o ritmo de jogo - 4, 5) em jogos disputados na fase regular e no playoff da liga portuguesa de Basquetebol(6). Os resultados obtidos pelos autores permitiram sugerir que, face à importância diferenciada dos jogos (os jogos do playoff são mais importantes), as equipas em confronto dispuseram de um menor número de posses de bola nos jogos do playoff, o que sugere um ritmo de jogo mais lento e, consequentemente, resultou em valores mais baixos de pontos marcados ${ }^{(6)}$. Este estudo acrescenta ainda que nestes jogos de playoff realizados a ritmos mais lentos, as equipas cometeram mais faltas, converteram mais lançamentos-livres e menos lançamentos de 2 pontos ${ }^{(6)}$. Neste domínio particular, as faltas cometidas, os ressaltos ofensivos, os lançamentos livres convertidos e falhados foram identificados como as estatísticas do jogo que melhor discriminam as equipas que vencem das equipas que perdem os jogos do playoff(5). Por outro lado, Sampaio(9) apresentou um estudo sobre o ritmo dos jogos da equipa do Leche Rio Breogán realizados durante a $1^{\mathrm{a}}$ e a $2^{\mathrm{a}}$ voltas da fase regular da liga espanhola de Basquetebol (Liga ACB). Neste trabalho, foram definidos dois grupos de jogos (mais rápidos e mais lentos) através de uma análise de classificação automática (cluster analysis) e analisaram-se as posses de bola e a eficácia ofensiva em função dos grupos resultantes dessa análise estatística prévia. Os resultados encontrados evidenciaram uma diminuição do ritmo da $1^{a}$ para a $2^{\mathrm{a}}$ volta (menor número de posses de bola) e, simultaneamente, um aumento na percentagem de vitórias sempre que o jogo decorria de forma mais lenta (valores superiores na eficácia ofensiva). Acrescentese ainda que a estatística do jogo que discriminou cada tipo de jogos (mais rápidos vs. mais lentos) foram os lançamentos de 3 pontos convertidos(9). Este estudo foi realizado com uma amostra de jogos disputados numa fase regular e sempre pela mesma equipa. Neste sentido, a validade de generalização dos resultados é limitada a esse mesmo contexto estratégico-táctico e daqui decorre a necessidade de confirmar estes resultados noutros contextos de análise mais diversificados e abrangentes.

Neste quadro operativo, o objectivo do presente trabalho é caracterizar o ritmo dos jogos de Basquetebol e identificar as estatísticas que melhor discriminam os jogos de ritmo mais elevado dos 
jogos de ritmo mais lento. Este tipo de informação poderá constituir-se de elevada importância para que as equipas se preparem melhor para este tipo de confrontos.

\section{METODOLOGIA}

\section{Amostra}

Os dados utilizados neste estudo foram recolhidos através dos registos estatísticos oficiais das três competições europeias por clubes (Euroliga, ULEBCUP e FIBA CUP). Foram utilizados os registos do jogo final de cada competição em cada época desportiva, o que constituiu uma amostra total de 27 jogos (Quadro 1). Esta opção foi tomada devido à elevada importância atribuída aos jogos finais, dado que o vencedor se torna campeão europeu. Neste sentido, é provável que os cuidados a ter na preparação destas competições sejam incrementados e que aumente a importância que se coloca nas decisões dos treinadores e dos jogadores.

Quadro 1. Distribuição da amostra por competição disputada e caracterização da diferença pontual final dos jogos.

\begin{tabular}{lccc}
\hline Competição & Épocas Desportivas & $\mathrm{n}$ & $\begin{array}{c}\text { Diferença pontual } \\
(\mathbf{x} \pm \mathbf{s d} \text { ) }\end{array}$ \\
\hline Euroliga & $1987-88$ a $2005-06$ & 19 & $7 \pm 4,5$ \\
ULEBCUP & $2002-03$ a 2005-06 & 5 & $8 \pm 4,7$ \\
FIBACUP & $2003-04$ a 2005-06 & 3 & $18 \pm 7,5$ \\
\hline TOTAL & $1987-88$ a 2005-06 & 27 & $8 \pm 5,4$ \\
\hline
\end{tabular}

\section{Procedimentos}

As estatísticas dos jogos foram recolhidas por técnicos especializados da FIBA (Fédération Internationale de Basketball) e consistiram no registo de frequências das seguintes acções técnico-tácticas: lançamentos de 2 e 3 pontos (convertidos e falhados), lançamentos livres (convertidos e falhados), ressaltos defensivos e ofensivos, assistências, roubos de bola, desarmes de lançamento, perdas de bola, faltas cometidas e sofridas. Posteriormente, todas as estatísticas recolhidas foram divididas pelos valores das posses de bola do jogo, no sentido de as normalizar à medida padrão de 100 posses de bola e assim garantir que a análise posterior dos dados não sofre contaminação do ritmo de jogo(4). Depois de normalizadas, todas as estatísticas de jogo foram multiplicadas por 100(5).
Considerou-se que uma equipa tem a posse da bola quando tem um controlo ininterrupto e completo da bola. As posses de bola, que representam o ritmo do jogo, foram calculadas através da seguinte equação(4):

Posses de bola $=$ (lançamentos de campo tentados) - (ressaltos ofensivos) + (perdas de bola) $-0,4 \times$ (lançamentos livres tentados)

Os lançamentos tentados representam o número total de vezes que os jogadores de uma equipa executam uma tentativa para lançar ao cesto, que terminará com sucesso ou insucesso.

Segundo este conceito, considera-se a conquista do ressalto ofensivo não como uma nova posse de bola mas como um "reavivar" da posse de bola anterior. Desta forma, poderemos constatar que no final dos jogos, as equipas em confronto, usufruíram aproximadamente do mesmo número de posses de bola, uma vez que uma equipa não pode dispor de posses de bola consecutivas ${ }^{(4)}$.

\section{Tratamento dos dados}

No sentido de dar resposta ao objectivo formulado, o tratamento dos dados iniciou-se pela identificação de jogos outliers de acordo com a diferença pontual final. De seguida procedeu-se à análise de clusters onde se constituíram 2 grupos de jogos, em função do número de posses de bola do jogo. Estes dois grupos foram categorizados como jogos mais rápidos e jogos mais lentos. Posteriormente passou-se à análise da função discriminante no sentido de identificar, através dos coeficientes canónicos estruturais (CCE) superiores a $|0,30|$, as estatísticas do jogo que mais contribuem para separar os jogos mais lentos dos jogos mais rápidos. A análise estatística foi realizada através do software estatístico SPSS versão 13.0 e o nível de significância foi mantido em $5 \%$.

\section{RESULTADOS}

Na Figura 1 encontram-se os resultados da variação do número de posses de bola, pontos marcados e eficácia ofensiva das finais europeias realizadas entre 1988 e 2006. Nestes resultados pode-se identificar uma tendência para a diminuição da eficácia ofensiva das equipas desde a época de 2002. 


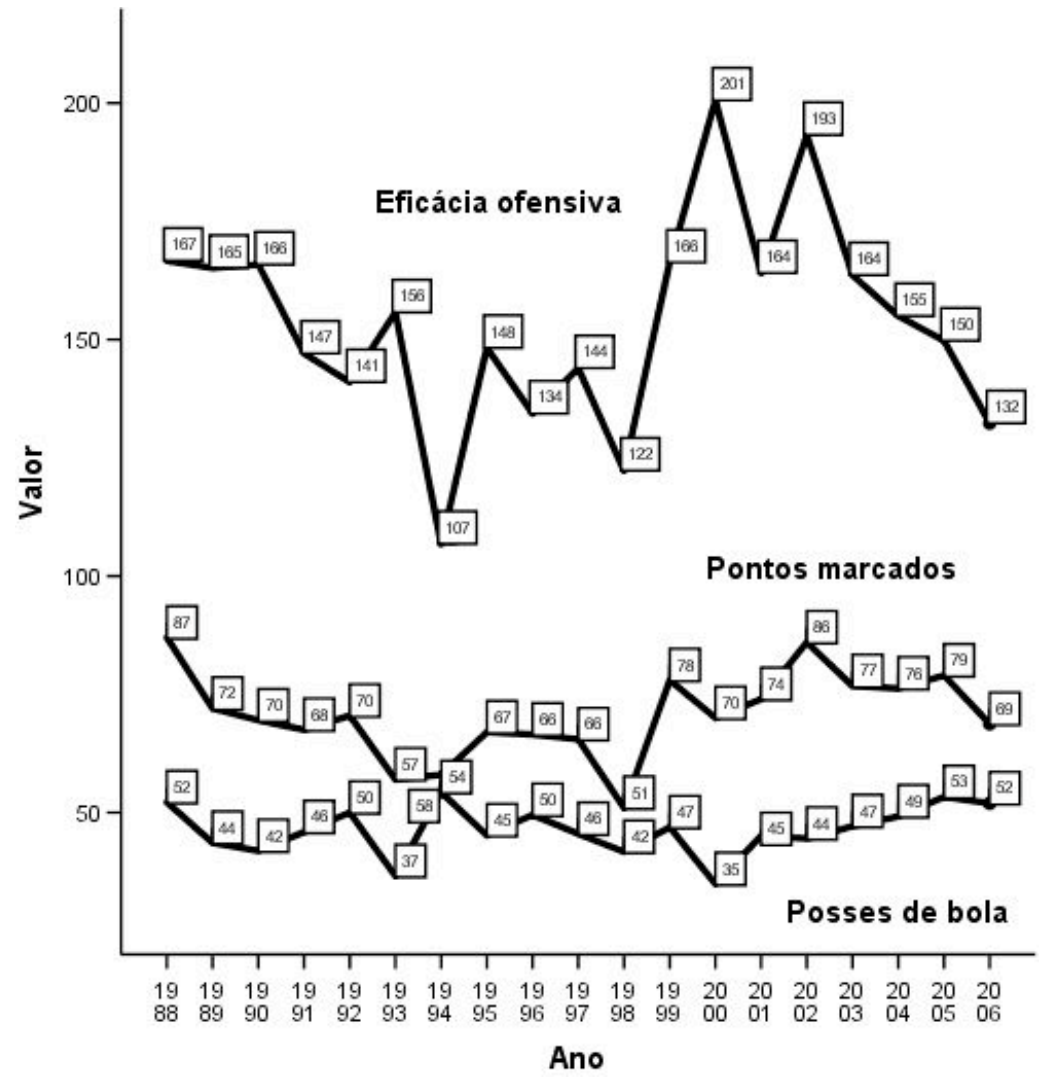

Figura 1. Variação do número de posses de bola, pontos marcados e eficácia ofensiva desde 1988 até 2006
Os valores médios de cada grupo de jogos produzidos pela análise de classificação automática foram para os jogos mais rápidos de $49,4 \pm 5,02$ posses de bola e para os jogos mais lentos de $40,5 \pm 5,63$ posses de bola. As diferenças na eficácia ofensiva das equipas destes dois contextos foram estatisticamente significativas, com valores de $149 \pm 19$ nos jogos mais rápidos e de $167 \pm 28$ nos jogos mais lentos. No Quadro 2 encontram-se os valores médios das estatísticas dos jogos para cada grupo de jogos (mais rápidos e mais lentos), bem como os resultados da comparação de médias.

Quadro 2. Médias e desvios-padrão dos indicadores estatísticos dos jogos mais rápidos e jogos mais lentos.

\begin{tabular}{|c|c|c|}
\hline Estatísticas dos jogos & $\begin{array}{c}\text { Jogos mais rápidos } \\
{[45,1 \leq \mathrm{PB} \leq 54,4]}\end{array}$ & $\begin{array}{c}\text { Jogos mais lentos } \\
{[34,9 \leq \mathrm{PB}<45,1]}\end{array}$ \\
\hline Lançamentos de 2 pontos Convertidos & $37,95 \pm 09,14$ & $43,93 \pm 09,88$ \\
\hline Lançamentos de 2 pontos Falhados & $42,19 \pm 11,66$ & $46,00 \pm 10,42$ \\
\hline Lançamentos de 3 pontos Convertidos & $12,49 \pm 04,58$ & $12,15 \pm 05,09$ \\
\hline Lançamentos de 3 pontos Falhados & $22,58 \pm 10,83$ & $25,27 \pm 09,21$ \\
\hline Lançamentos Livres Convertidos & $35,75 \pm 13,55$ & $42,84 \pm 17,79$ \\
\hline Lançamentos Livres Falhados* & $12,82 \pm 06,12$ & $19,33 \pm 09,46$ \\
\hline Ressaltos Defensivos* & $40,53 \pm 10,06$ & $50,27 \pm 10,86$ \\
\hline Ressaltos Ofensivos & $22,46 \pm 09,63$ & $24,54 \pm 06,38$ \\
\hline Assistências & $22,93 \pm 11,17$ & $18,86 \pm 06,75$ \\
\hline Roubos de Bola & $16,52 \pm 07,80$ & $17,82 \pm 10,12$ \\
\hline Desarmes de Lançamento & $5,46 \pm 05,39$ & $5,28 \pm 05,15$ \\
\hline Perdas de Bola & $26,57 \pm 08,06$ & $22,06 \pm 07,26$ \\
\hline Faltas Sofridas* & $49,40 \pm 08,98$ & $61,04 \pm 15,30$ \\
\hline Faltas Cometidas* & $49,36 \pm 08,97$ & $61,04 \pm 15,30$ \\
\hline
\end{tabular}


A função discriminante obtida para diferenciar os jogos mais lentos dos jogos mais rápidos foi estatisticamente significativa $(p \leq 0,05)$. Os CCE mais relevantes para o compósito linear desta função foram os das faltas cometidas e sofridas, dos ressaltos defensivos e dos lançamentos livres falhados (ver Quadro 3).

Quadro 3. Estatísticas do jogo que discriminam os jogos mais rápidos dos jogos mais lentos.

\begin{tabular}{lc}
\hline Estatísticas dos jogos & CCE \\
\hline Faltas Sofridas* & 0,44 \\
Faltas Cometidas* & 0,43 \\
Ressaltos Defensivos* & 0,38 \\
Lançamentos Livres Falhados* & 0,37 \\
Lançamentos de 2 pontos Convertidos & 0,26 \\
Perdas de Bola & $-0,23$ \\
Lançamentos Livres Convertidos & 0,19 \\
Assistências & $-0,16$ \\
Lançamentos de 2 pontos Falhados & 0,13 \\
Lançamentos de 3 pontos Falhados & 0,10 \\
Ressaltos Ofensivos & 0,09 \\
Roubos de Bola & 0,06 \\
Lançamentos de 3 pontos Convertidos & $-0,03$ \\
Desarmes de Lançamento & $-0,01$ \\
& \\
Wilks' Lambda & 0,46 \\
Qui-Quadrado & 31,82 \\
$P$ & $<0,05$ \\
Eigenvalue & 1,17 \\
Correlação Canónica & 0,74 \\
\hline
\end{tabular}

${ }^{*}|C \subset \varepsilon| \geq 0,30$

\section{DISCUSSÃO}

O objectivo do presente trabalho foi caracterizar o ritmo dos jogos de Basquetebol e identificar as estatísticas que melhor discriminam os jogos de ritmo mais elevado dos jogos de ritmo mais lento. Existem opiniões de que os jogos mais lentos são característicos das fases mais decisivas das épocas desportivas, como por exemplo os jogos da $2^{a}$ volta da fase regular (que decidem a classificação para os playoffs, ou a manutenção na divisão) e os jogos dos playoffs (que decidem a continuação ou não na luta pelo título de campeão ou pelo acesso às competições europeias) $(6,9)$.
Os valores de corte apresentados para o presente estudo que permitiram diferenciar os jogos disputados a ritmos lentos dos jogos disputados a ritmos rápidos, devem ser entendidos no contexto particular da amostra estudada. De facto, os valores médios obtidos para os jogos da presente amostra são substancialmente inferiores aos obtidos em estudos que recorreram à mesma metodologia realizados noutros contextos $(4,9)$. Contudo, estes valores sugerem que todos os jogos da presente amostra decorreram a ritmos mais lentos, facto que confirma as opiniões previamente apresentadas.

$\mathrm{Na}$ literatura científica internacional, estes assuntos têm sido tratados exclusivamente em jogos da Liga Profissional Norte-Americana (NBA) por Oliver(4). Neste diferente contexto, o autor identificou um aumento na eficácia ofensiva e uma diminuição nas posses de bola e nos pontos marcados por jogo. Grosso modo, os resultados encontrados no presente estudo parecem distintos das opiniões apresentadas e dos estudos disponíveis, porque sugerem uma tendência recente para a diminuição da eficácia ofensiva e dos pontos marcados. Por outro lado, o número de posses de bola por jogo tem aumentado. O sentido de variação deste conjunto de macro-estatísticas do jogo de Basquetebol sugere que os jogos das finais mais recentes são disputados a ritmos mais elevados, mas com prejuízo nos pontos marcados e na eficácia ofensiva. O facto destes jogos serem decisivos para a atribuição dos títulos de campeão europeu, provavelmente reflectir-se-á numa percepção diferente do jogo pelos jogadores, que lhe atribuirão mais importância porque todas as acções que executem podem ser decisivas para o resultado final do confronto $(3,7)$. Provavelmente, este é um aspecto que se vai reflectir mais no seu desempenho ofensivo, onde é necessária mais concentração na execução das estratégias colectivas e na conversão de lançamentos $(10,12,13)$. Em caso contrário, podem ocorrer selecções de lançamento pouco favoráveis e com poucas possibilidades de conquista do ressalto ofensivo. É neste sentido que se verifica a superioridade das defesas sobre os ataques, levando à consequente diminuição dos pontos marcados e ao aumento do número de posses de bola por jogo. Este aumento das posses de bola não é necessariamente positivo para as equipas pois, no presente estudo, foram 
identificadas diferenças estatisticamente significativas entre o ritmo dos jogos e a eficácia ofensiva, com valores superiores para os jogos mais lentos (jogos mais rápidos $149 \pm 19 v s$. jogos mais lentos $167 \pm 28$ ). Neste sentido, tentar disputar um destes jogos finais de forma mais lenta, explorando constantemente as situações de ataque organizado de modo a colocar os jogadores nas suas posições de melhor eficácia individual de lançamento e de maiores possibilidades de disputa dos ressaltos ofensivos, pode ajudar a obter melhores eficácias ofensivas. Neste assunto particular, a literatura sugere que, nestes momentos, se explorem os jogadores que desempenham funções mais próximo do cesto(11, 12). Por outro lado, também se diminui as hipóteses da equipa adversária explorar situações de transição defesa-ataque em superioridade numérica, caracterizadas pelas elevadas probabilidades de sucesso $(5,8,14)$.

No que diz respeito à identificação das estatísticas que melhor discriminam os jogos de ritmo mais elevado $(45,1 \leq$ posses de bola $\leq 54,4)$ dos jogos de ritmo mais lento $(34,9 \leq$ posses de bola $<45,1)$, os resultados do presente estudo identificaram as faltas (cometidas e sofridas), os ressaltos defensivos e os lançamentos livres falhados como as estatísticas que melhor discriminaram estes dois contextos.

Alguns estudos $(9,10)$ indicam que o aumento da frequência e do poder discriminante do número de faltas cometidas e de lançamentos livres tem caracterizado estes jogos decisivos onde o ritmo é mais lento. Sampaio(9) refere o número de lançamentos de 3 pontos convertidos como o indicador estatístico que mais diferencia os jogos mais lentos dos mais rápidos.

$O$ poder discriminante das faltas sugere que o ritmo de jogo pode ser imposto (acelerado ou desacelerado) pelo recurso a esta estratégia. Ou seja, as equipas que participaram nestes confrontos recorreram às faltas, como meio de impedir a realização de lançamentos fáceis, por exemplo parar as situações de superioridade numérica do adversário, parar as recepções de bola em espaços de elevada eficácia ofensiva (i.e., a zona mais próxima do cesto habitualmente ocupada pelos postes baixos) e mesmo parar jogadores de elevada eficácia ofensiva(11). Desta forma, aumenta o número de faltas cometidas e sofridas nestes jogos e diminui o seu ritmo. Esta situação implica, também, um aumento do número de lançamentos livres (quer seja como consequência das faltas em acto de lançamento, quer seja como resultado do acumular de faltas). O aumento do número de lançamentos livres tentados e a importância de converter pontos nestes jogos, é uma explicação provável para o aumento do número de lançamentos livres falhados. Este facto, pode favorecer a conquista da posse de bola por parte da equipa defensora.

Os resultados do presente estudo realçam igualmente o poder discriminante dos ressaltos defensivos. Este aspecto pode resultar do facto de ocorrerem mais lançamentos livres falhados, o que aumenta as possibilidades de conquistar mais ressaltos, em especial da equipa que está a defender (devido à vantagem posicional da defesa no lançamento livre). Em suma, os jogos das finais das competições europeias de Basquetebol estão a decorrer a ritmos mais elevados, mas com menor eficácia e menos pontos marcados. O ritmo dos jogos é essencialmente controlado pelas faltas cometidas e sofridas, facto que também é a expressão da estratégia preconizada pelos treinadores.

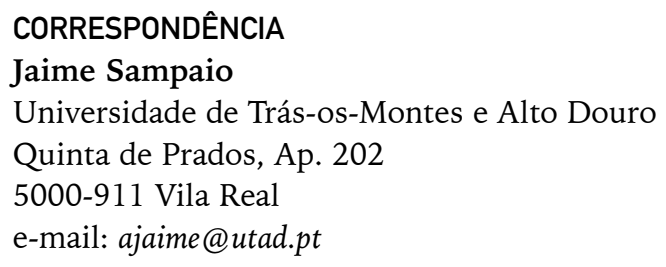




\section{BIBLIOGRAFIA}

1. De Rose D (2004). Statistical analysis of basketball performance indicators according to home/away games and winning and losing teams. Journal of Human Movement Studies 47: 327-336.

2. Ibanez SJ, Sampaio J, Saenz-Lopez P, Gimenez J, Janeira MA (2003). Game statistics discriminating the final outcome of Junior World Basketball Championship matches (Portugal 1999). Journal of Human Movement Studies 45: 119.

3. Kozar B, Whitfield KE, Lord RH, Mechikoff RA (1993). Timeouts before Free-Throws - Do the statistics support the strategy. Perceptual and Motor Skills 76: 47-50.

4. Oliver D (2004). Basketball On Study: Rules and Tools for Performance Analysis. Dulles: Brassey's, Inc.

5. Sampaio J, Janeira MA (2003) Statistical Analyses of Basketball Team Performance: Understanding Teams' Wins and Losses According to a Different Index of Ball Possessions. International Journal of Performance Analysis in Sport 1: 40-49.

6. Sampaio J, Janeira MA (2002). A Vantagem Casa nos Jogos da Liga Profissional de Basquetebol: Diferenças entre os Jogos da Fase Regular e os Jogos do Playoff. In: Janeira MA, Brandão E (eds.), Estudos 3 CEJD. Porto: FCDEF-UP, 93100.

7. Sampaio J, Janeira MA (2003). Importance of Free-Throw Performance in Game Outcome during the Final Series of Basketball Playoffs. International Journal of Applied Sports Science 2: 9-16.
8. Sampaio J, Janeira MA (2005). A Vantagem Casa nos Jogos Desportivos Colectivos: Revisão da Literatura Centrada no Basquetebol e no Modelo de Courneya e Carron. Revista Portuguesa de Ciências do Desporto 2: 235-246.

9. Sampaio J (2003). Análise da Eficácia Colectiva ao Longo do Jogo de Basquetebol: Perspectivas Transversais e Longitudinais Centradas nos Resultados de uma Equipa de Alto Nível. In: Ibáñez S, Mercedes M (eds.). Actas de las ponências invitadas del $2^{\circ}$ Congresso Ibérico de baloncesto., Cáceres: Facultad de Ciencias del Deporte de la Universidad de Extremadura.

10. Sampaio J, Ibáñez SJ, Feu S (2004). Discriminative Power of Basketball Game-Related Statistics by Level of Competition and Sex. Perceptual and Motor Skills 32: 1231-1238.

11. Sampaio J, Janeira MA, Ibáñez S, Lorenzo A (2006). Discriminant analysis of game-related statistics between basketball guards, forwards and centres in three professional leagues. European Journal of Sport Science 6: 163-178.

12. Trninic S, Dizdar D, Dezman B (2002). Pragmatic validity of the combined model of expert system for assessment and analysis of the actual quality overall structure of basketball players. Collegium Antropologicum 26: 199-210.

13. Trninic S, Dizdar D, Luksic E (2002). Differences between winning and defeated top quality basketball teams in final tournaments of European club championship. Collegium Antropologicum 26: 521-531.

14. Wang YT, Chen SH, Limroongreungrat W, Change LS (2005). Contributions of selected fundamental factors to wheelchair basketball performance. Medicine and Science in Sports and Exercise 37: 130-137. 